\title{
A New Computational Approach to Photon-Assisted Tunneling in Intense Driving Fields Based on a Fabry-Perot Analogy
}

\author{
MATHIAS WAGNER \\ Hitachi Cambridge Laboratory, Madingley Road, Cambridge CB3 OHE, United Kingdom
}

\begin{abstract}
Quantum transport in multiple-barrier systems exposed to intense laser fields is investigated by employing an analogy with the Fabry-Perot interferometer of classical optics. This analogy is exact if no further approximations are introduced. The FabryPerot approach is shown to be quite robust: Approximating the barriers by $\delta$-functions with equivalent dc transmission and reflection amplitudes still yields excellent agreement with "exact" orthodox transfer-matrix calculations. The advantage of the former is its much reduced computational demands as well as ease of use. $73.40 . \mathrm{Gk}, 73.50 . \mathrm{Pz}$, 72.40. $+\mathrm{w}, 42.50 . \mathrm{Hz}$
\end{abstract}

Keywords: Tunneling, quantum transport, time-dependent fields, laser, Fabry-Perot

\section{INTRODUCTION}

It is commonly agreed that ultimately, with the ever increasing miniaturization of devices, one will have to resort to quantum-transport theories for a proper modeling of device characteristics. On the other hand, it is equally clear that today's quantum-transport theories are less mature than semi-classical approaches [1] and demand huge computer resources in order to give only reasonably accurate results. Moreover, most quantumtransport theories currently available are restricted to steady-state operations or a small-signal analysis. Although strict theoretical treatments of nonequilibrium Green's functions with arbitrary timedependence and initial many-particle correlations do exist [2], they are generally too complicated to be easily implemented. Nevertheless, serious efforts are being made in this direction (See, for instance, the NEMO project described in [3]).

The issue we want to address in this paper is that of quantum transport in the presence of intense external time-dependent driving fields where the usual small-signal analysis fails. The subject of (in) coherent quantum transport in strongly driven heterostructures has recently gained much momentum with the advent of free-electron lasers, which are capable of providing intense radiation [4]. Here we report on a new approach to the notoriously complex problem of a theoretical description of quantum transport in intense laser fields that is based on an analogy with the Fabry- 
Perot interferometer in optics. In previous studies, a transfer-matrix method had been employed to calculate the reflection and transmission amplitudes of scattering states in driven heterostructures [5-10]. Due to the large size of the transfer matrix even for a one-dimensional system (the size being proportional to the highest order of photon processes one wishes to take into account), these calculations demand large computer resources when aimed at studying the regime of strong driving. In contrast, the Fabry-Perot approach is rather modest in its computational demands and moreover, much faster for not too complex heterostructures. Our aim here is not to develop a fully-fledged theory for realistic device simulation that includes all possible scattering mechanisms, but rather to put forward a useful tool for gaining a good understanding of the basic physics involved in the quantum-transport in strongly driven systems. To this end we seek to solve the time-dependent single-particle Schrödinger equation but refrain from making any assumptions whatsoever about the strength of the driving field. The additional degree of freedom introduced by an explicit time dependence of the Hamiltonian opens up the possibility for new effects to be explored, only a few of which have already been discovered and studied in some detail, such as the "dynamic localization" of minibands in driven superlattices $[11,12]$ and the "quenching of tunneling" in driven tunneling diodes $[6,8]$.

\section{FABRY-PEROT THEORY}

The textbook approach to calculating the probability for an electron to tunnel through a multiple-barrier structure is to solve for the corresponding scattering state with the proper boundary conditions, which consist of a traveling incoming wave and reflected as well as transmitted outgoing waves. A convenient way of doing this is to set up and solve a transfer-matrix problem [6,8]. There is, however, an alternative approach that takes advantage of an analogy with optics [13]: Rather than solving a large system of simultaneous equations, one can track the electron in a manner resembling ray tracing: An electron incident on the first barrier has some probability to tunnel. If it does tunnel, it will travel to the next barrier, where it may be reflected or again tunnel. However, the electron reflected at the second barrier is not lost, it still has a chance to tunnel through this barrier. All it needs to do is to travel back to the first barrier, get reflected there and come back to the second barrier to have a second try. It is clear that in a multiple-barrier structure the number of possible paths an electron can take and still finally arrive at the far side is enormous. However, all these paths can be summed up in a few geometric series as we will demonstrate in the following.

Before proceeding any further, however, let us introduce the notation pertinent to tunneling in driven systems. The main difference compared to ordinary tunneling in static structures is that in a harmonically driven system the electron can pick up or lose integer units of photon quanta $\hbar \omega$ from the driving field. Hence, the electron is not restricted to its original energy $E$, but rather has a finite probability of being found in one of the photonic sidebands or "channels" at energies $E+n \hbar \omega$. As a consequence, partial transmission probabilities will need to be calculated for all channels individually. Obviously, the number of sidebands to take into account for this will depend on the strength of the driving field.

Let us denote the partial transmission amplitude of a single driven barrier from channel $j$ (i.e., with incident energy $E_{j}$ ) to channel $l$ as $t_{l j}^{l, r}$, where the superscripts $l, r$ indicate from which side the electron impinges on the barrier (see Fig. 1). A similar notation is used for the reflection: $r_{l j}^{l, r}$. Furthermore, the "optical" path across a quantum well of width $d$ carries the phase factor $Q_{l j}=\delta_{l j}$ $\exp \left(i k_{l} d\right)$ with $k_{l}=\sqrt{2 m\left(E_{0}+l \hbar \omega\right)} / \hbar$ being the wave vector of sideband $l$.

For a double-barrier diode in particular, the transmission amplitudes for an electron incident, 


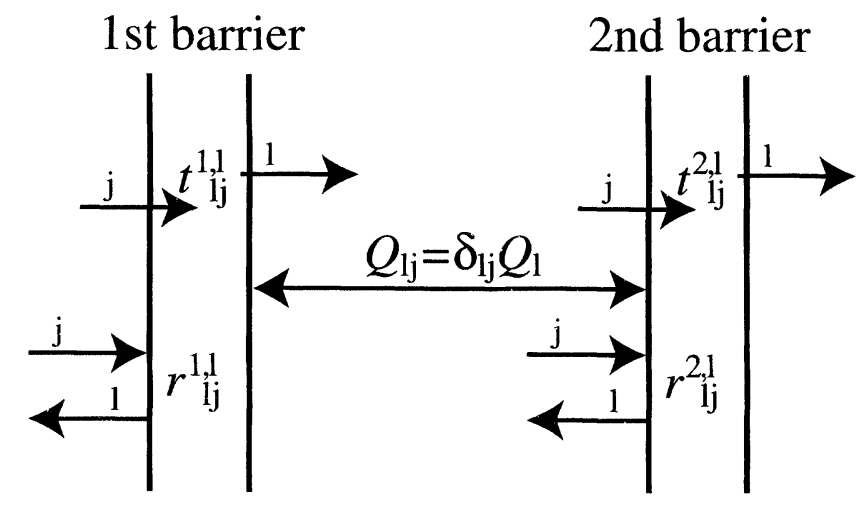

FIGURE 1 Schematics of the notations used for the transmission and reflection amplitudes and the optical path $Q$ in the quantum well that underlie the Fabry-Perot approach.

say, from the left-hand side can be summed up in a geometric series, which in a shorthand matrix notation can be written as

$$
t=t^{2, l}\left(I+R+R^{2}+\cdots\right) Q t^{1, l}=t^{2, l}(I-R)^{-1} Q t^{1, l},
$$

with $I$ being the identity matrix and the kernel $R=$ $Q r^{1, r} Q r^{2, l}$ describing one reflection cycle in the quantum well starting and ending at the left wall of the right barrier. Note that while in the static case $R$ is a scalar, it is a matrix of infinite rank for finite ac driving. In a similar fashion, the reflection amplitudes at the double-barrier diode are found as

$$
r=r^{1, l}+t^{1, r} Q r^{2, l}(I-R)^{-1} Q t^{1, l} .
$$

To give the reader an impression of the complexity to expect when treating more than two barriers, we also present the formula for the transmission amplitude through a triple barrier diode,

$$
\begin{aligned}
t= & t^{3, l} \\
& \times \frac{1}{I-\frac{1}{\left(I-R_{2}\right)} Q_{2} t^{2, l} \frac{1}{\left(I-R_{1}\right)} Q_{1} r^{1, r} Q_{1} t^{2, r} Q_{2} r^{3, l}} \\
& \times \frac{1}{\left(I-R_{2}\right)} Q_{2} t^{2, l} \frac{1}{\left(I-R_{1}\right)} Q_{1} t^{1, l},
\end{aligned}
$$

where the reciprocal is understood to denote the matrix inverse.
As usual, the transmission probability is obtained from the transmission amplitude via $T_{j l}=$ $\left.\left|k_{j}\right| k_{l}\left|m_{l} / m_{j}\right| t_{j l}\right|^{2}$. The main advantage of a formulation along these lines is that the basic building blocks of reflection and transmission amplitudes through single barriers may be determined by other means, either approximately or even exactly. For instance, assuming the photon energy to be small compared to the energy of the incident electron, the transmission and reflection amplitudes through a single $\delta$-function barrier, driven by a uniform ac field $F \cos \omega t$, are approximately given by [13]

$$
\begin{aligned}
& t_{n}=t_{\mathrm{dc}} i^{n} \delta_{n 0}, \\
& r_{n}=r_{\mathrm{dc}}(-i)^{n} J_{n}(2 a),
\end{aligned}
$$

where $a=e F k_{0} / m \omega^{2}$, with $k_{0}$ being the wave vector of the incident electron, $J_{n}$ the $n$th Bessel function of the first kind and $t_{\mathrm{dc}}$ and the $r_{\mathrm{dc}}$ the transmission and reflection amplitudes, respectively, for the static barrier. In this approximation, the kernel $R$ of Eqs. (1) and (2) describing the multiple reflections in the quantum well is evaluated as

$$
\begin{gathered}
R_{j l}=r_{\mathrm{dc}}^{1, r} r_{\mathrm{dc}}^{2, l} \sum_{n} \exp \left(i k_{j} d\right) i^{j-l-n} J_{j-l-n}(2 a) \\
\times \exp \left(i k_{l+n} d\right)(-i)^{n} J_{n}(2 a) \\
\approx r_{\mathrm{dc}}^{1,} r_{\mathrm{dc}}^{2, l} \exp \left\{i k_{0} d[2+v(3 j+l) / 4]\right\} \\
\times J_{j-l}\left(\left|4 a \sin \left(k_{0} d v / 4\right)\right|\right)
\end{gathered}
$$


where $v=\hbar \omega / E_{0}$ is the dimensionless photon energy.

\section{RESULTS FOR A DOUBLE-BARRIER DIODE}

For the remainder of this paper we shall focus on results for double-barrier diodes driven by a spatially uniform ac field $F \cos \omega t$. Although the Fabry-Perot method by itself is exact, the subsequent approximations made above such as the assumption of $\delta$-barriers etc. may affect the final result. However, our findings indicate that the agreement with the standard transfer-matrix technique [10] is still excellent. Figure $2 \mathrm{a}$ presents typical results of the Fabry-Perot approach, based on Eqs. (1) and (4-5). Shown is the probability for an electron incident at the energy $E_{0}$ of the undriven resonance, to transverse a symmetric double-barrier structure and emerge at the far side in sideband $n$, i.e., at energy $E_{0}+n \hbar \omega$. To leading order, the sideband amplitudes depend on the dimensionless parameter $a=e F k_{0} / m \omega^{2}$. For comparison, we also present in Figure $2 b$ the results of a calculation using transfer matrices. The agreement is very good, with minor discrepancies appearing in the asymmetry of the "mirror" sidebands at $n= \pm|n|$, which are due to the approximations introduced in Eqs. (4-5). Apart from being computationally faster by one or two orders of magnitude compared to the transfermatrix method, a major virtue of the Fabry-Perot approach lies in the fact that for generating Figure $2 \mathrm{a}$ we have only used the $d c$ transmission and reflection amplitudes of a single, undriven barrier. This is a substantial advantage, as it allows us to "plug in" the transmission and reflection amplitudes obtained by using other approaches. Incidentally, the fact that the entire effect of the driving ac field can be subsumed in the Bessel function factors of Eq. (4) is a rather surprising result by itself. And last but not least, the FabryPerot approach is more amenable to introducing a phase-breaking mechanism by randomizing the phase in the optical-path matrix $Q$ traversing the quantum well [14].

The agreement between the Fabry-Perot and the transfer-matrix method is just as good for other energies of the incident electron. In Figure 3 we assumed all parameters to be the same as in Figure 2, except for the electron's energy, which we took to be equal to that of the $n=-1$ sideband, i.e., $E=E_{0}-\hbar \omega$. Most noteworthy in this Figure is the exact reproduction of the splitting of the $n=0$ and $n=2$ channels for small values of $e F k_{0} /$ $m \omega^{2}$, which cannot be accounted for in a simpler product ansatz proposed earlier [6].

The pronounced dips in the transmission probability seen in Figures 2 and 3 at particular values of the driving field are a manifestation of the

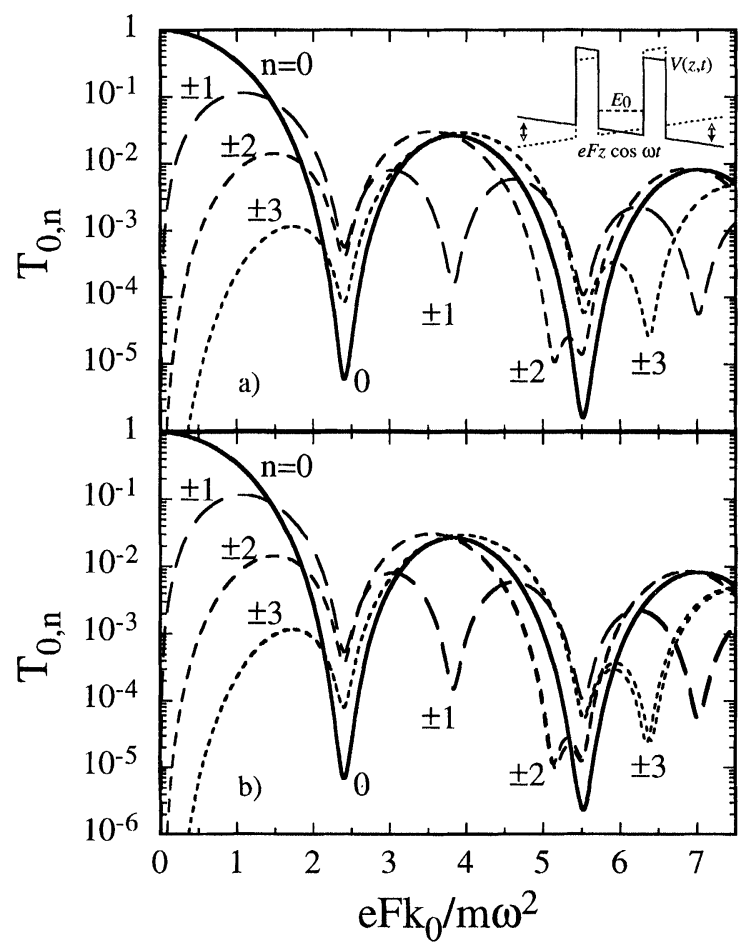

FIGURE 2 Transmission probability in the lowest few photonic sidebands through a driven double-barrier diode (see inset in top panel) as a function of driving strength for an electron incident at energy $E_{0}$, the energy of the lowest resonance in the static quantum well: a) Fabry-Perot, b) transfer-matrix method. 


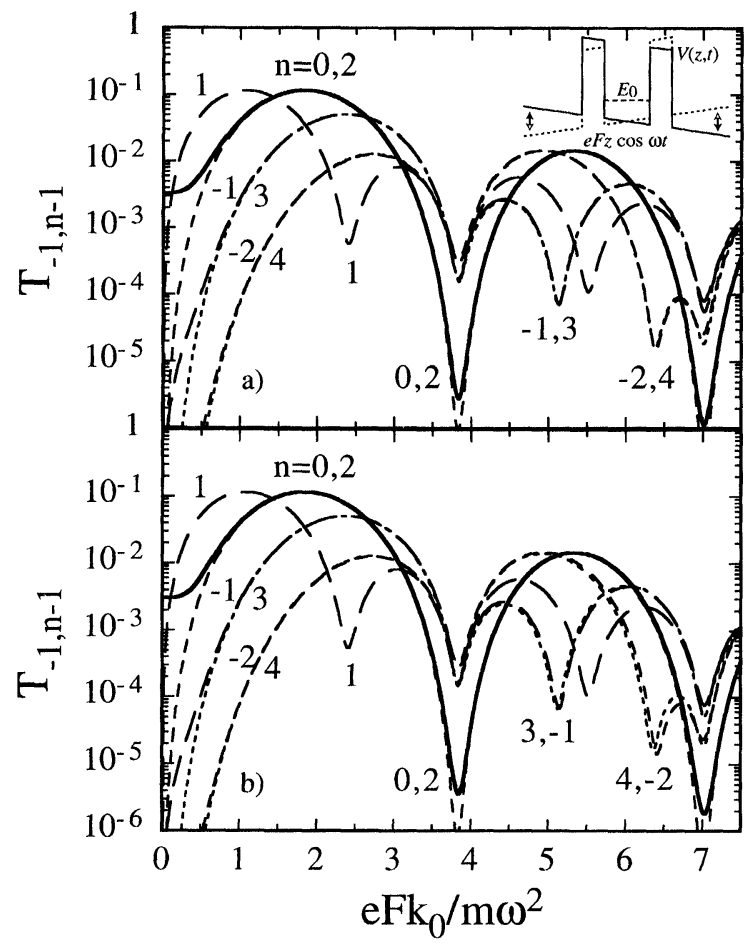

FIGURE 3 Same as Figure 2, but for an electron incident at energy $E_{0}-\hbar \omega$, i.e., one photon quantum below the quantumwell resonance.

"quenching of tunneling" predicted for strongly driven systems [6]. Basically, in order to traverse the double-barrier structure, an incident electron first has to couple into the driven resonance in the quantum well. The efficiency for this to happen is proportional to the overlap of the electronic wave function with that of the driven resonance. A similar overlap integral occurs when the electron exits the quantum well. Evidently, the electron cannot couple to the entire wave function of the driven resonance, but rather interacts only with its energy component or "spectral weight" at the energy of the electron. This spectral weight turns out to be a strong function of the driving field [9] and in particular may have pronounced minima under suitable driving conditions, which leave their fingerprints as characteristic minima in the transmission probability.

\section{CONCLUSIONS}

In conclusion, a Fabry-Perot approach has been used to reduce the problem of coherent transmission and reflection in strongly driven complex tunneling structures to that of single driven barriers. This approach gives valuable insight into the physics involved and is easy to use at the same time. It is even possible to go one step further by replacing the finite-height barriers with $\delta$ barriers having the same effective dc transmission and reflection amplitudes. It then turns out that the ac driving field affects only the relative amplitudes of the photonic sidebands, while the dc transmission and reflection probabilities determine the absolute magnitude summed over all sidebands - even for finite ac fields.

\section{References}

[1] Ferry, D. (1997). "Open problems in quantum simulation in ultradeep-submicron devices", Fifth International Workshop on Computational Electronics, Notre Dame, Indiana, May 28-30

[2] Wagner, M. (1991). "Expansions of non-equilibrium Green's functions", Phys. Rev. B, 44, 6104.

[3] Klimeck, G. et al. (1995). "Quantum device simulation with a generalized tunneling formula", Appl. Phys. Lett., 67, 2539.

[4] Keay, B. J. et al. (1995). "Photon-assisted electric field domains and multiphoton-assisted tunneling in semiconductor superlattices", Phys. Rev. Lett., 75, 4098.

[5] Liu, H. C. (1988). "Time-dependent approach to doublebarrier quantum well oscillators", Appl. Phys. Lett., 52, 453.

[6] Wagner, M. (1994). "Quenching of resonant transmission through an oscillating quantum well", Phys. Rev. B, 49, 16544.

[7] Iñarrea, J., Platero, G. and Tejedor, C. (1994). "Coherent and sequential photoassisted tunneling through a semiconductor double-barrier structure", Phys. Rev. B, 50, 4581 .

[8] Wagner, M. (1995). "Photon-assisted transmission through an oscillating quantum well: A transfer-matrix approach to coherent destruction of tunneling", Phys. Rev. A, 51, 798 .

[9] Wagner, M. (1996). "Strongly driven quantum wells: An analytical solution to the time-dependent schrödinger equation", Phys. Rev. Lett., 76, 4010.

[10] Wagner, M. (1996). "Coherent destruction of tunneling in resonant tunneling diodes driven by a strong laser field", in Quantum Coherence and Decoherence, Foundations of Quantum Mechanics in the Light of New Technology, edited by K. Fujikawa and Y. A. Ono (Elsevier, Amsterdam), p. 147. 
[11] Holthaus, M. (1992). "The quantum theory of an ideal superlattice responding to far-infrared laser radiation", Z. Phys. B, 89, 251.

[12] Holthaus, M. (1992). "Collapse of minibands in farinfrared irradiated superlattices", Phys. Rev. Lett., 69, 351.

[13] Wagner, M. and Zwerger, W. (1997). "Characteristic scaling parameters for tunneling in strong time-dependent electric fields", Phys. Rev. B, 55, 10217R.

[14] $\mathrm{Hu}, \mathrm{Y}$. (1988). "An optical model of double-barrier resonant tunneling oscillators", J. Phys. C, 21, L23.

\section{Author Biography}

Mathias Wagner received his Diploma in 1988 and his Dr. rer. nat. in 1990 in Physics, both from
Hamburg University, Germany, where he was also awarded the membership of the Studienstiftung des Deutschen Volkes. Except for $11 / 2$ years at the Central Research Laboratory, Hitachi Ltd., Tokyo, he has since been working in the Hitachi Cambridge Laboratory, UK, where his main interests include quantum transport in driven systems and correlated electronic systems. He is a member of the German Physical Society. 

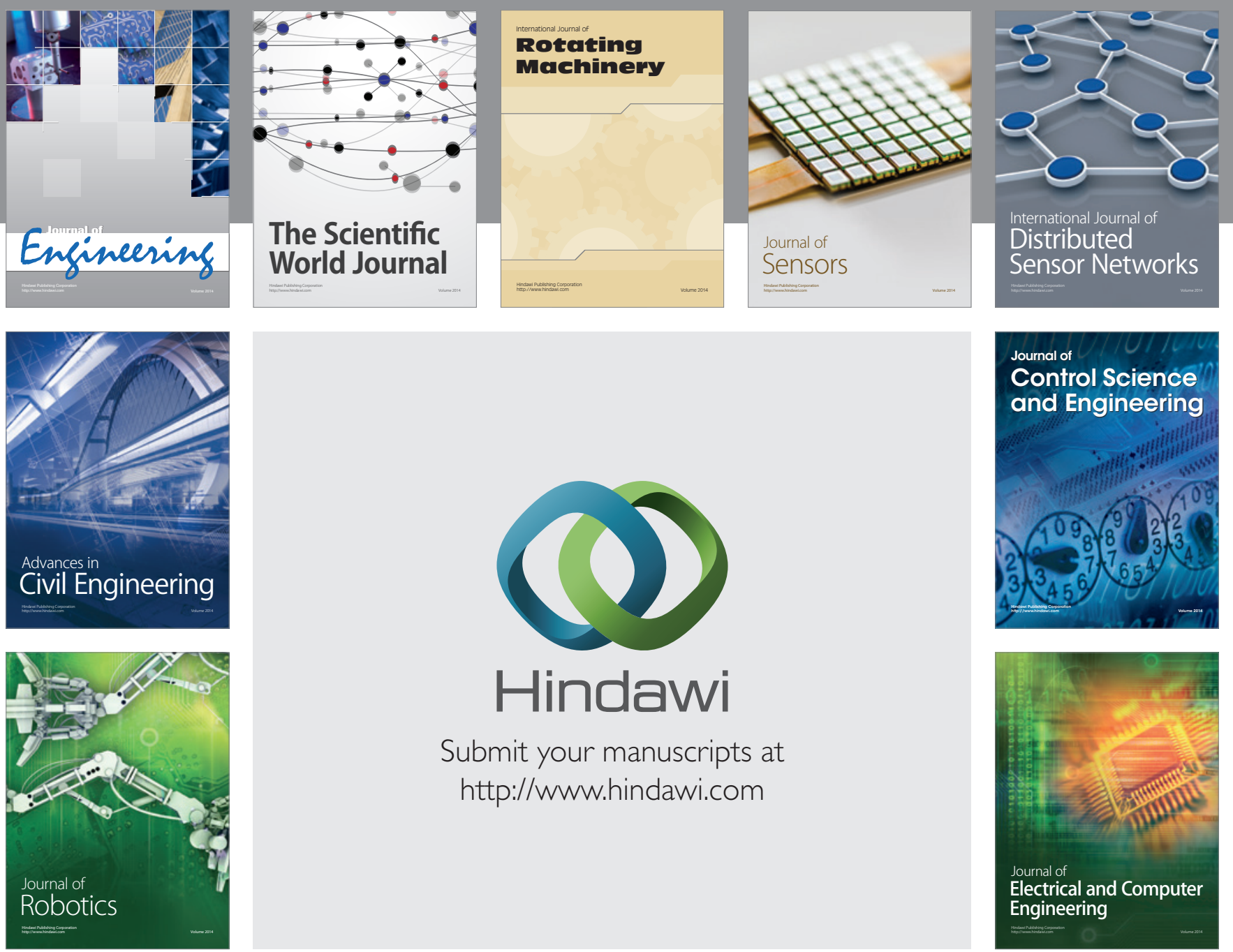

Submit your manuscripts at

http://www.hindawi.com
